\title{
Mecanismos de desactivación de catalizadores heterogéneos
}

\section{Deactivation mechanisms of heterogeneous catalysts}

\author{
J. G. Téllez-Romero, ** P. Sarabia-Bañuelos, ${ }^{* *}$ S. Hernández-González, * \\ V. A. Nolasco-Arizmendi*
}

\begin{abstract}
Catalytic deactivation is a serious problem in different sections of the oil refining process, causing loss of catalytic activity with respect to operating time. This review is focused on the deactivation catalyst mechanisms, such as poisoning, fouling, thermal degradation and sintering, chemical degradation and mechanical failures like attrition and crushing, in fluid catalytic cracking (FCC), hydrodesulfurization (HDS), and catalytic reforming. The causes of these deactivation catalyst mechanisms are chemical, thermal and mechanical. The key features and considerations for each of these deactivation mechanisms types are reviewed. Additionally, the total cost for catalyst deactivation is increasing gradually each year because of the replacement of spent catalyst, generating thousands of tons of industrial waste.
\end{abstract}

KEYWORDS: heterogeneous catalyst, deactivation mechanisms, catalytic deactivation.

RESUMEN: La desactivación catalítica es un problema serio en las diferentes secciones del proceso de refinación del petróleo, que causa la pérdida de actividad catalítica con respecto al tiempo de operación. La presente revisión está enfocada en los mecanismos de desactivación de catalizadores, tales como envenenamiento, ensuciamiento, degradación térmica y sinterización, degradación química y fallas mecánicas como el desgaste y el aplastado del catalizador, en el craqueo catalítico fluidizado (FCC), la hidrodesulfuración (HDS) y el reformado catalítico. Las causas de estos mecanismos de desactivación catalítica son químicos, térmicos y mecánicos. Se revisan las características y consideraciones clave para cada uno de estos tipos de mecanismos de desactivación. Además, el costo total por la desactivación catalítica aumenta gradualmente cada año debido al remplazo del catalizador gastado, generando miles de toneladas de desechos industriales.

PALABRAS CLAVE: catalizador heterogéneo, mecanismos de desactivación, desactivación catalítica.

\section{Introducción}

El proceso de refinación del petróleo tiene como finalidad transformar compuestos de bajo valor comercial a productos de alto valor comercial para sa-

Recibido: 10 de marzo de 2020.

Aceptado: 13 de julio de 2020.

\footnotetext{
* Universidad Tecnológica de Tula-Tepeji, Química Industrial.

** Universidad Nacional Autónoma de México, Facultad de Química.

- Autor de correspondencia: tellez_gabriel@hotmail.com
} 
tisfacer las necesidades de la sociedad en cuestiones de energía, transporte, salud y bienestar. Para ello, es necesario el empleo de procesos químicos que transformen la materia prima (petróleo) mediante el empleo de catalizadores heterogéneos. Por ejemplo, tres de los principales procesos catalíticos empleados en la refinación del petróleo son:

1. El proceso de craqueo catalítico, conocido como FCC (por sus siglas en inglés: fluid catalytic cracking), es uno de los procesos más importantes del esquema de refinación por la capacidad de transformar las fracciones pesadas del petróleo en gasolinas de alto octanaje, a través de reacciones de desintegración catalítica, contribuyendo así al pool de gasolinas. La desintegración catalítica se realiza con el empleo de catalizadores heterogéneos ácidos, donde el componente activo es un aluminosilicato con estructura cristalina denominada zeolita, responsable de la selectividad de los productos. Las zeolitas empleadas en el proceso FCC son: zeolita X, zeolita Y y ZSM-5. Las zeolitas $\mathrm{X}$ e $\mathrm{Y}$ tienen la misma estructura cristalina, pero las primeras tienen una relación sílice/alúmina menor y una menor estabilidad térmica que las segundas. Por tal motivo, en la actualidad el proceso FCC utiliza la zeolita $\mathrm{Y}$ o algunas variaciones de esta, tales como la zeolita USY y REY; mientras que la ZSM-5 es una zeolita versátil que incrementa tanto el rendimiento de las olefinas ligeras como el número de octano (Félix Flores, 2006). Una mejora realizada a las zeolitas tradicionales del proceso de FCC es la adición de tierras raras como lantano (La), que incrementa la actividad catalítica (Liu et al., 2014). Recientemente, nuevas zeolitas han sido probadas como catalizadores en el proceso de FCC, remplazando a las zeolitas tradicionales, tal es el caso de las zeolitas ITQ-7, ITQ-17, ITQ-21, ITQ-33, ITQ-39, ZSM20, entre otras (Bai et al., 2018; Castañeda et al., 2006; Vogt et al., 2015). Dichas zeolitas presentan estructuras porosas tridimensionales, adquiriendo propiedades como fuerza ácida similar o mayor a la zeolita ZSM-5 así como una alta estabilidad térmica (Castañeda et al., 2006; Vogt et al., 2015). La demanda mundial de catalizadores de FCC ha incrementado drásticamente de 10,000 ton/año en 1996 a 90,000 ton/año en 2014 (Fuentes, 1996; Vogt et al., 2015).

2. El hidrotratamiento (HDT) es otro de los procesos más importantes dentro de la industria de la refinación del petróleo. El objetivo de este proceso es reducir la cantidad de compuestos contaminantes provenientes de las fracciones de destilado de petróleo crudo, tales como: compuestos orgánicos con $\mathrm{S}, \mathrm{N}, \mathrm{O}$, compuestos aromáticos, y metales como $\mathrm{Ni}, \mathrm{Na}, \mathrm{Fe}, \mathrm{V}$, por mencionar algunos, los cuales afectan negativamente las propiedades de rendimiento de los productos terminados (gasolinas, diésel), pues durante la combustión de los automóviles disminuyen la calidad del aire por la presencia de com- 
puestos orgánicos volátiles (COV), NOx, y SOx. Los catalizadores empleados en el HDT son sulfuros de Ni-Mo, Co-Mo, y Ni-W, soportados en $\gamma-\mathrm{Al}_{2} \mathrm{O}_{3}$. Es por ello que el hidrotratamiento es empleado para cumplir con las regulaciones ambientales de los hidrocarburos, las cuales son cada vez más estrictas. La demanda mundial de este tipo de catalizador fue de 100,000 a 120,000 ton/año entre los años 2002 y 2007 (Martino y Boitiaux, 2002; Dufresne, 2007).

3. El proceso de reformado catalítico tiene como propósito fundamental incrementar el número de octano de la nafta obtenida en la destilación atmosférica. Este proceso emplea catalizadores bimetálicos de metales nobles, tales como Pt-Re o Pt-Ir, ambos soportados en $\gamma-\mathrm{Al}_{2} \mathrm{O}_{3}$. Las reacciones químicas que se llevan a cabo son: deshidrogenación, isomerización, deshidrociclización, aromatización, entre otras (Zakari-Yusuf et al., 2019). La demanda mundial de catalizadores de reformado catalítico fue de 6,000 ton/año en 2002, lo que representa un costo de $€ 20 / \mathrm{kg}$ (Martino y Boitiaux, 2002).

Por lo que se refiere a nivel mundial, el consumo industrial de catalizadores heterogéneos fue de 800,000 ton/año en 2012, generando ventas superiores a $\$ 163,000$ millones de dólares (Li et al., 2016).

Uno de los principales inconvenientes presentes durante el empleo de catalizadores heterogéneos industriales es la desactivación inminente e inevitable con el transcurso del tiempo de los sitios activos, acompañada de la pérdida de actividad catalítica y disminución de la selectividad del producto deseado (Zhou et al., 2020). Por ejemplo: a) el catalizador del proceso FCC se desactiva por la formación de coque depositado sobre la superficie del catalizador (Al-Khattaf, 2002; Bai et al., 2018; Ino et al., 1996); b) el proceso de desactivación de los catalizadores de hidrodesulfuración (HDS) se realiza en tres fases: una desactivación rápida debido a la formación de coque en la superficie del catalizador; una desactivación lenta debida al recubrimiento de la superficie de los catalizadores por metales, como $\mathrm{Ni}, \mathrm{Va}, \mathrm{Na}$, entre otros, y, una desactivación rápida causada por el bloqueo de los poros del catalizador debida a la deposición de metales y coque (Kallinikos et al., 2008; Seki et al., 2001). Aunado a esto, el proceso HDT genera un subproducto azufrado de reacción, el $\mathrm{H}_{2} \mathrm{~S}$, el cual debe ser eliminado antes de entrar en otras etapas del proceso de refinación, tal es el caso del proceso de hidrogenación para incrementar el número de cetano del diésel, ya que el $\mathrm{H}_{2} \mathrm{~S}$ desactiva fácilmente los catalizadores de hidrogenación a base de metales como el Ni y metales nobles, Pt y Pd (Song, 1999; Song y Schmitz, 1997). c) Los catalizadores empleados en el proceso de reformado catalítico son desactivados por la deposición de coque sobre la superficie del catalizador (Zakari Yusuf et al., 2019).

Los tres procesos anteriores descritos brevemente tienen algo en común: la desactivación del catalizador empleado por diversos mecanismos. En general, la desactivación del catalizador es la pérdida de la actividad o selecti- 
vidad catalítica presentada por los catalizadores heterogéneos conforme transcurre el tiempo de operación (Argyle y Bartholomew, 2015; Bartholomew et al., 1982). La desactivación del catalizador se atribuye a cualquier interacción física o química entre el catalizador y las impurezas presentes en la corriente de proceso, las cuales bloquean los sitios activos o compiten con los reactantes para ocupar dichos sitios. En el caso de los catalizadores de FCC se desactivan por la formación de coque en la superficie externa o en las bocas de los poros.

Por ello, el propósito del presente artículo es proporcionar información científica y aspectos teóricos sobre la disminución o pérdida de la actividad catalítica enfocada en los mecanismos de desactivación de catalizadores heterogéneos y los factores que participan en ella. Además, la desactivación de la fase activa, promotor o soporte de los catalizadores, inicia por la formación e interacción física o química de contaminantes sobre la superficie del catalizador; así como también la sinterización de las partículas metálicas de escala nanométrica, las cuales son las responsables de la disminución de la actividad catalítica.

\section{Mecanismos de desactivación}

En varios procesos químicos más de un mecanismo de desactivación limita la vida útil del catalizador, generando periodos cortos de operación, que van desde unos cuantos minutos hasta 15 años de funcionamiento. Es por ello que se tiene un gran interés en incrementar la vida útil del catalizador en aquellas fases donde el tiempo es corto o donde existe desactivación irreversible; así como también estudiar los mecanismos involucrados en la desactivación de catalizadores.

En la tabla 1 se muestran algunas reacciones químicas industriales, el tipo de catalizador, el tiempo de vida útil, los procesos de desactivación involucrados y las propiedades afectadas del catalizador (Argyle y Bartholomew, 2015).

Los mecanismos de desactivación de los catalizadores se pueden clasificar en las siguientes categorías (Argyle y Bartholomew, 2015; Larsson, 2007; Zhou et al., 2020):

1. Envenenamiento.

2. Ensuciamiento.

3. Degradación térmica y sinterización.

4. Degradación química.

5. Fallas mecánicas.

\section{Desactivación por envenenamiento}

El envenenamiento es la quimisorción fuerte de reactivos, productos o impurezas sobre sitios activos disponibles para llevar a cabo la reacción. Para 
TABLA 1. Tiempo de vida útil de catalizadores y condiciones de operación de algunos procesos químicos.

\begin{tabular}{|c|c|c|c|c|c|}
\hline Reacción & Catalizador & $\begin{array}{l}\text { Tiempo de } \\
\text { vida (años) }\end{array}$ & $\begin{array}{l}\text { Condiciones } \\
\text { de operación }\end{array}$ & $\begin{array}{l}\text { Proceso de } \\
\text { desactivación }\end{array}$ & $\begin{array}{l}\text { Propiedad } \\
\text { catalítica } \\
\text { afectada }\end{array}$ \\
\hline $\begin{array}{c}\text { Craqueo } \\
\text { catalítico de } \\
\text { aceites }\end{array}$ & $\begin{array}{l}\text { Zeolitas } \\
\text { (lecho } \\
\text { fluidizado) }\end{array}$ & 0.000002 & $\begin{array}{c}500-560{ }^{\circ} \mathrm{C} \\
2-3 \mathrm{~atm}\end{array}$ & $\begin{array}{l}\text { Formación } \\
\text { rápida de } \\
\text { coque }\end{array}$ & $\begin{array}{c}\text { Actividad } \\
\text { Transferencia } \\
\text { de masa }\end{array}$ \\
\hline $\begin{array}{c}\text { Reformación } \\
\text { catalítica }\end{array}$ & $\mathrm{Pt} / \mathrm{Al}_{2} \mathrm{O}_{3}$ & $0.01-0.5$ & $\begin{array}{l}490-530^{\circ} \mathrm{C} \\
10-25 \text { atm }\end{array}$ & $\begin{array}{l}\text { Formación de } \\
\text { coque }\end{array}$ & $\begin{array}{c}\text { Actividad } \\
\text { Transferencia } \\
\text { de masa }\end{array}$ \\
\hline $\begin{array}{l}\text { Oxidación de } \\
\text { amoniaco }\end{array}$ & Pt-Rh & $0.1-0.5$ & $\begin{array}{c}800-900{ }^{\circ} \mathrm{C} \\
1-10 \mathrm{~atm}\end{array}$ & $\begin{array}{l}\text { Rugosidad de la } \\
\text { superficie } \\
\text { Pérdida de Pt }\end{array}$ & $\begin{array}{c}\text { Selectividad } \\
\text { Ensuciamiento }\end{array}$ \\
\hline $\begin{array}{l}\text { Reducción de } \\
\text { aldehídos a } \\
\text { alcoholes }\end{array}$ & $\mathrm{Cu} / \mathrm{ZnO}$ & $0.5-1$ & $\begin{array}{c}220-270^{\circ} \mathrm{C} \\
100-300 \\
\text { atm }\end{array}$ & $\begin{array}{c}\text { Sinterización } \\
\text { lenta }\end{array}$ & Actividad \\
\hline $\begin{array}{l}\text { Steam } \\
\text { reforming de } \\
\text { gas natural }\end{array}$ & $\mathrm{Ni} / \alpha-\mathrm{Al}_{2} \mathrm{O}_{3}$ & $1-3$ & $\begin{array}{c}500-850{ }^{\circ} \mathrm{C} \\
30 \text { atm }\end{array}$ & $\begin{array}{l}\text { Sinterización } \\
\text { Envenenamiento } \\
\text { Formación de } \\
\text { carbón }\end{array}$ & Actividad \\
\hline $\begin{array}{c}\text { Sintesis de } \\
\text { metanol }\end{array}$ & $\mathrm{Cu} / \mathrm{ZnO}-\mathrm{Al}_{2} \mathrm{O}_{3}$ & $2-5$ & $\begin{array}{l}200-300{ }^{\circ} \mathrm{C} \\
50-100 \text { atm }\end{array}$ & $\begin{array}{c}\text { Sinterización } \\
\text { lenta } \\
\text { Envenenamiento } \\
\text { por } \mathrm{Sy} \mathrm{Cl}\end{array}$ & Actividad \\
\hline $\begin{array}{l}\text { Hidrogenación } \\
\text { de acetileno }\end{array}$ & Pd soportado & $5-10$ & $\begin{array}{l}30-150{ }^{\circ} \mathrm{C} \\
20-30 \text { atm }\end{array}$ & $\begin{array}{l}\text { Sinterización } \\
\text { lenta }\end{array}$ & $\begin{array}{c}\text { Actividad } \\
\text { Selectividad }\end{array}$ \\
\hline $\begin{array}{l}\text { Síntesis de } \\
\text { amoniaco }\end{array}$ & $\begin{array}{l}\text { Fe, promotor: } \\
\mathrm{K}_{2} \mathrm{O} \text { y } \\
\text { estabilizador: } \\
\qquad \mathrm{Al}_{2} \mathrm{O}_{3}\end{array}$ & $10-15$ & $\begin{array}{c}450-470{ }^{\circ} \mathrm{C} \\
200-300 \mathrm{~atm}\end{array}$ & $\begin{array}{l}\text { Sinterización } \\
\text { lenta }\end{array}$ & Actividad \\
\hline
\end{tabular}

Fuente: Tomada de Argyle y Bartholomew (2015).

que una especie química actúe como un contaminante, depende de su fuerza de adsorción en comparación con otras especies químicas que compiten por los mismos sitios catalíticos. Además, los contaminantes adsorbidos pueden inducir cambios en la estructura electrónica o geométrica de la superficie. Dependiendo de la fuerza de adsorción, los catalizadores presentan dos tipos de desactivación: reversible e irreversible. Un ejemplo de desactivación reversible es la desactivación de los sitios ácidos de los catalizadores del proceso FCC, los cuales son regenerados mediante el incremento de la temperatura y la introducción de una corriente de aire. Sin embargo, la mayoría de los compuestos contaminantes son sustancias que se quimisorben irreversiblemente a la superficie de los sitios activos, como tal es el caso del catalizador de Ni soportado y desactivado por la quimisorción de azufre.

Uno de los contaminantes ampliamente estudiado es el átomo de azufre, el cual se encuentra en forma de heterocompuestos orgánicos como tiofeno, 
benzotiofeno, dibenzotiofeno y 4,6-dimetildibenzotiofeno o en forma de compuesto inorgánico como sulfuro de hidrógeno. Con el fin de estudiar la desactivación de los catalizadores de hidrogenación de etileno debido al envenenamiento causado por azufre, se han realizado modelos conceptuales de dos dimensiones. Al principio, un átomo de azufre fuertemente adsorbido bloquea físicamente al menos cuatro sitios activos de reacción. Posteriormente, debido al enlace metal-azufre fuertemente formado, el azufre modifica electrónicamente los átomos metálicos más cercanos y los átomos metálicos próximos más cercanos, modificando la capacidad de adsorción y/o disociación de las moléculas reactantes $\left(\mathrm{H}_{2}\right.$ y etileno). Como resultado de la adsorción fuerte metal-azufre, la superficie metálica se restructura causando cambios dramáticos en las propiedades catalíticas del metal. Además, los átomos de azufre fuertemente adsorbidos disminuyen la difusión superficial de las moléculas reactantes adsorbidas, tal como se observa en la figura 1.

Estudios previos de adsorción indican que el $\mathrm{H}_{2} \mathrm{~S}$ se adsorbe fuerte y disociativamente sobre la superficie metálica del níquel. La alta estabilidad y baja reversibilidad del azufre adsorbido se representa en una gráfica de equilibrio, donde se grafica $\log \left(\mathrm{P}_{\mathrm{H} 2 \mathrm{~S}} / \mathrm{P}_{\mathrm{H} 2}\right)$ versus el recíproco de la temperatura. La mayoría de la información de adsorción se encuentra entre $\Delta \mathrm{H}$ que va de -125 y $-165 \mathrm{~kJ} / \mathrm{mol}$ para valores de 0.5 a 0.9 de cobertura de la superficie metálica por el azufre. Esto indica que el azufre adsorbido es más estable que el bulk $\mathrm{Ni}_{3} \mathrm{~S}_{2}$. De hecho, la extrapolación de datos a alta temperatura a cobertura cero produce una entalpía de adsorción de $-250 \mathrm{~kJ} / \mathrm{mol}$. En otras palabras, a bajas coberturas, los enlaces $\mathrm{Ni}-\mathrm{S}$ superficial tienen un

FIGURA 1. Modelo conceptual de la desactivación por envenenamiento causado por azufre de los sitios metálicos durante la hidrogenación de etileno.

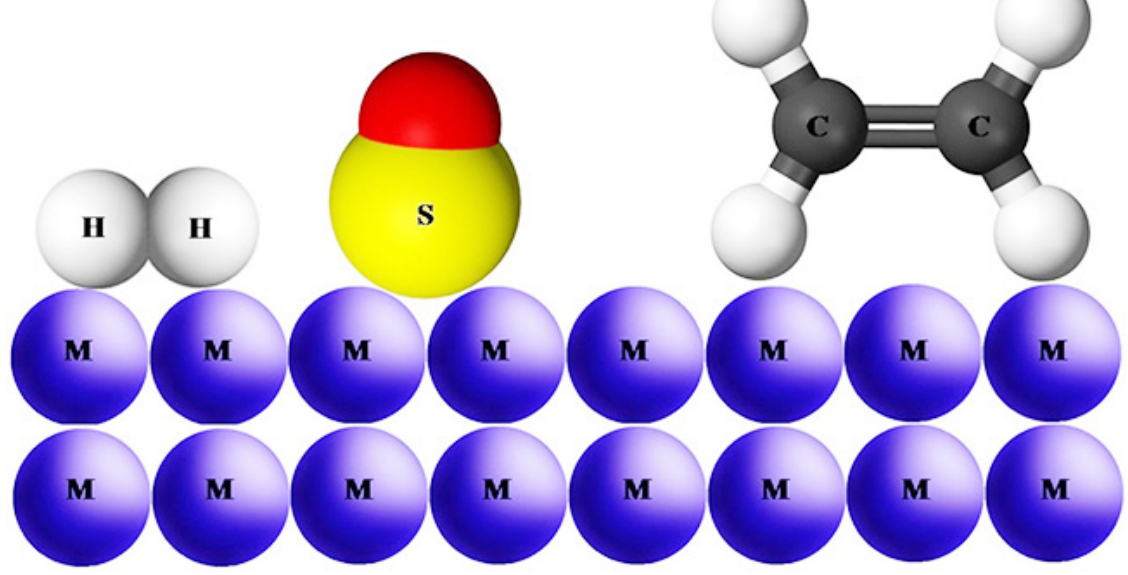

Fuente: Tomado de Argyle y Bartholomew (2015). 
factor de tres veces más estabilidad que los enlaces presentes en el compuesto $\mathrm{Ni}_{3} \mathrm{~S}_{2}$.

Es evidente que la presión parcial de equilibrio de $\mathrm{H}_{2} \mathrm{~S}$ aumenta con el incremento de la temperatura, aumentando la cobertura. Por ejemplo, a $450{ }^{\circ} \mathrm{C}$ $(725 \mathrm{~K})$ y $\theta=0.5$, el valor de $\mathrm{P}_{\mathrm{H} 2 \mathrm{~S}} / \mathrm{P}_{\mathrm{H} 2}$ va de $1 \times 10^{-8}$ a $1 \times 10^{-9}$. En otras palabras, la mitad del recubrimiento de la superficie metálica por átomos de azufre ocurre con 1 - 10 ppb $\mathrm{H}_{2} \mathrm{~S}$, que es un intervalo de concentración muy bajo. A la misma temperatura, $450{ }^{\circ} \mathrm{C}$, el recubrimiento de los átomos de azufre es mayor a 0.9 a valores de $\mathrm{P}_{\mathrm{H} 2 \mathrm{~S}} / \mathrm{P}_{\mathrm{H} 2}$ que va de $1 \times 10^{-7}$ a $1 \times 10^{-6}$, lo equivalente a 0.1 - 1 ppm $\mathrm{H}_{2} \mathrm{~S}$ o a concentraciones presentes en muchos procesos catalíticos después de que el gas ha sido eliminado. Por lo cual, se podría esperar que el $\mathrm{H}_{2} \mathrm{~S}$ (o cualquier otra impureza de azufre) se adsorberá esencialmente de una forma irreversible con una cobertura total de la superficie metálica en la mayoría de los procesos que involucren catalizadores metálicos (Bartholomew et al., 1982).

Con el fin de amortiguar la pérdida de actividad catalítica en la hidrogenación a causa de la presencia de compuestos azufrados, se han preparado catalizadores bimetálicos a base de Ni-Pt (Tellez-Romero et al., 2015) o Pt-Pd (Yasuda y Yoshimura, 1997) soportados. Otro parámetro estudiado para mejorar la hidrogenación de moléculas en presencia de compuestos azufrados es el empleo de soportes ácidos como las zeolitas (Song et al., 1997).

Otro átomo ampliamente estudiado es el átomo de nitrógeno, el cual puede formar moléculas orgánicas como piridina, pirrol, indol, quinolina, entre otras, o inorgánicas como amoniaco.

Además, existen otros átomos o compuestos que desactivan a los catalizadores, principalmente metálicos, los cuales se pueden clasificar de acuerdo con su origen químico y al tipo de interacción con el metal, tal como se observa en la tabla 2.

Así también, la tabla 3 enlista los contaminantes más comunes de los catalizadores de reacciones representativas importantes.

Además, otros términos significativos asociados con la desactivación de los catalizadores heterogéneos por el envenenamiento de átomos o moléculas se definen en la tabla 4.

TABLA 2. Clasificación de los contaminantes comunes de acuerdo con su estructura química.

\begin{tabular}{c|c|c}
\hline Tipo de contaminante & Ejemplos & Tipo de interacción con el metal \\
\hline V y VI A & $\mathrm{N}, \mathrm{P}, \mathrm{As}, \mathrm{Sb}, \mathrm{O}, \mathrm{S}, \mathrm{Se}, \mathrm{Te}$ & A través de los orbitales s y $p$ \\
\hline VII A & $\mathrm{F}, \mathrm{Cl}, \mathrm{Br}, \mathrm{I}$ & A través de los orbitales s y $p$ \\
\hline $\begin{array}{c}\text { Moléculas adsorbidas a través } \\
\text { de enlaces múltiples }\end{array}$ & $\begin{array}{c}\mathrm{CO}, \mathrm{NO}, \mathrm{HCN}, \\
\text { benceno, acetileno, otros } \\
\text { hidrocarburos insaturados }\end{array}$ & $\begin{array}{c}\text { Quimisorción a través de } \\
\text { enlaces múltipes y retroenlace }\end{array}$ \\
\hline Metales pesados tóxicos & $\mathrm{As}, \mathrm{Pb}, \mathrm{Hg}, \mathrm{Cd}$ & Ocupación de los orbitales $d$ \\
\hline
\end{tabular}

Fuente: Tomado de Argyle y Bartholomew (2015). 
Mundo Nano | ARTículos DE REVISıón | www.mundonano.unam.mx

14(26), 1e-16e, enero-junio 2021 | https:// doi.org/10.22201/ceiich.24485691e.2021.26.69642

J. G. Téllez-Romero, P. Sarabia-Bañuelos, S. Hernández-González, V. A. Nolasco-Arizmendi

TABLA 3. Contaminantes de catalizadores presentes en reacciones importantes.

\begin{tabular}{c|c|c}
\hline Catalizador & Reacción & Contaminante \\
\hline Sílica-alúmina, zeolitas & Cracking & $\begin{array}{c}\text { Bases orgánicas, } \\
\text { hidrocarburos y metales } \\
\text { pesados }\end{array}$ \\
\hline $\mathrm{Ni}, \mathrm{Pt}, \mathrm{Pd}$ & $\begin{array}{c}\text { Hidrogenación, } \\
\text { deshidrogenación }\end{array}$ & $\begin{array}{c}\text { Compuestos con azufre, } \mathrm{P}, \\
\mathrm{As}, \mathrm{Zn}, \mathrm{Hg}, \text { haluros, Pb, } \\
\mathrm{NH}_{3} \text { y C } \mathrm{H}_{2}\end{array}$ \\
\hline $\mathrm{Ni}$ & $\begin{array}{c}\text { Reformado de metano } \\
\text { con vapor }\end{array}$ & $\mathrm{H}_{2} \mathrm{~S}$ y As \\
\hline $\mathrm{Fe}, \mathrm{Ru}$ & Sintesis de amoniaco & $\mathrm{O}_{2}, \mathrm{H}_{2} \mathrm{O}, \mathrm{CO}, \mathrm{S}, \mathrm{C}_{2} \mathrm{H}_{2}$ \\
\hline $\mathrm{Co}, \mathrm{Fe}$ & Sintesis Fischer-Tropsch & $\begin{array}{c}\mathrm{H}_{2} \mathrm{~S}, \mathrm{As}, \mathrm{NH}_{3} \mathrm{y} \\
\text { carbonilos metálicos }\end{array}$ \\
\hline
\end{tabular}

Fuente: Tomado de Argyle y Bartholomew (2015).

TABLA 4. Parámetros empleados en la desactivación por envenenamiento de sitios catalíticos.

\begin{tabular}{c|l} 
Parámetro & \multicolumn{1}{c}{ Definición } \\
\hline Susceptibilidad & $\begin{array}{l}\text { Medida de la sensibilidad que presenta un catalizador hacia un } \\
\text { contaminante determinado. }\end{array}$ \\
\hline Toxicidad & $\begin{array}{l}\text { Susceptibilidad de un catalizador dado para un contaminante en relación } \\
\text { con otro contaminante. }\end{array}$ \\
\hline Resistencia & $\begin{array}{l}\text { Es el inverso de la velocidad de desactivación. Propiedad que determina qué } \\
\text { tan rápido un catalizador se desactiva. }\end{array}$ \\
\hline Tolerancia & $\begin{array}{l}\text { Actividad que presenta un catalizador cuando la superficie está saturada del } \\
\text { contaminante (algunos catalizadores presentan actividad insignificante). }\end{array}$
\end{tabular}

Fuente: Tomado de Argyle y Bartholomew (2015).

\section{Desactivación por ensuciamiento}

El ensuciamiento es la deposición física de sustancia sobre la superficie del catalizador provenientes de la fase fluida, ocasionando pérdida de actividad catalítica debido al bloqueo de los sitios activos o al bloqueo del sistema poros para acceder hasta el sitio activo. La desactivación de los catalizadores heterogéneos se debe a la formación de depósitos de carbón o coque sobre los sitios activos, donde el coque es producido por una serie de reacciones, tales como isomerización, oligomerización, alquilación, ciclización, aromatización, entre otras, formando hidrocarburos de alto peso molecular e incluso grafito (Hou et al., 2017). Los compuestos precursores de coque depositado en los catalizadores gastados del proceso FCC son principalmente hidrocarburos aromáticos de dos anillos presentes en la alimentación e hidrocarburos que presentan átomos de $\mathrm{S}, \mathrm{N}$ y $\mathrm{O}$ en su estructura, donde dichos compuestos reaccionan para forman compuestos poliaromáticos de tres o cuatro anillos aromáticos (Qian et al., 1997). Por otro lado, Appleby et al. (1962) identificaron que la formación 
de coque incrementa rápidamente si la alimentación presenta compuestos con anillos condensados, tales como naftaleno, antraceno y fenantreno, que sí presenta anillos enlazados, tales como bifenilo y terfenilo. Además, identificaron que la formación de coque se incrementa al aumentar la constante de basicidad de los compuestos aromáticos, obteniéndose cantidades de hasta $24 \%$ en peso de coque en el catalizador si la alimentación presenta antraceno.

Menon (1990) sugirió que las reacciones catalíticas acompañadas por formación de coque pueden ser clasificadas como sensibles o insensibles al coque. En las reacciones sensibles al coque, el coque no reactivo se deposita sobre los sitios activos, disminuyendo la actividad catalítica, tal es el caso del craqueo catalítico. Por otro lado, las reacciones insensibles al coque, donde el coque reactivo formado sobre los sitios activos es fácilmente removido por hidrógeno u otro agente gasificante, por ejemplo, la síntesis de FischerTropsch, el reformado catalítico y la síntesis de metanol.

Con el fin de visualizar los efectos que la formación de carbón genera en los catalizadores de metales soportados, se han presentado diversos modelos conceptuales de dicho comportamiento, tal es el caso del modelo conceptual de ensuciamiento mostrado por Bartholomew en la figura 2 (Bartholomew et al., 2006).

Este modelo conceptual muestra las siguientes características:

a) El coque se quimisorbe fuertemente como una monocapa o se adsorbe físicamente en multicapas, bloqueando el acceso de moléculas reactantes a los sitios catalíticos.

b) Encapsulamiento total de las partículas metálicas, obteniendo desactivación completa.

c) Bloqueo de los microporos y mesoporos.

FIGURA 2. Modelo conceptual de ensuciamiento.

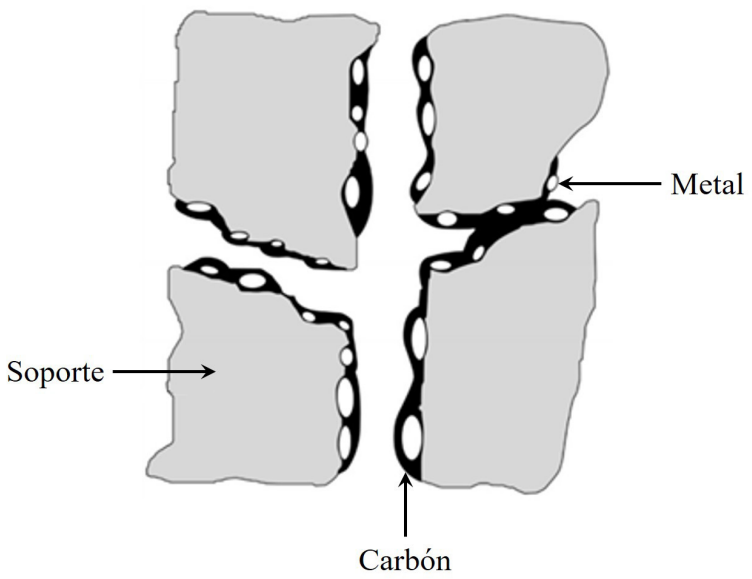

Fuente: Tomado de Bartholomew et al. (2006). 
Generalmente, la velocidad de formación de coque es función de la acidez y de la estructura porosa del soporte, es decir, la velocidad de formación de coque es directamente proporcional a la fuerza ácida y al tamaño de los poros. Por ejemplo, con el catalizador de zeolita $\mathrm{Y}$ de diámetro de poro de $0.72 \mathrm{~nm}$, se tiene un contenido de coque del $2.2 \%$ en peso, mientras que con el catalizador ZSM- 5 de diámetro de poro $0.54 \times 0.56 \mathrm{~nm}$, solo $0.4 \%$ en peso de coque (Naccache, 1985). Ambos catalizadores son ampliamente utilizados en el proceso FCC. Furimsky y Massoth (1999) concluyeron que la actividad catalítica disminuye si se emplean tamaños de poros pequeños, esto debido al bloqueo de las bocas de los poros; mientras que, a tamaños de poros más grandes, la pérdida de la actividad catalítica es a causa de la desactivación de los sitios activos internos del catalizador, llamando a este modelo como core poisoning.

En el caso de los catalizadores bifuncionales (metal/óxido metálico), se forman diferentes tipos de coque: coque blando depositado en la fase metálica y coque duro depositado sobre el soporte (Augustine et al., 1989). Los precursores de coque se forman por la generación de radicales libres que, posteriormente, experimentan reacciones de polimerización, causando disminución de la actividad catalítica isomerizante del soporte. Mackor et al. (1958) estudiaron la generación de radicales libres a partir de los compuestos que se encuentran presentes en la corriente de alimentación, tales como benceno, naftaleno, bifenilo, fenantreno, antraceno, naftaceno, trifenileno, criseno, pireno, entre otros.

Otro caso ampliamente estudiado se presenta en los catalizadores de hidrotratamiento, estos muestran una rápida desactivación debida a la deposición de coque y a la adsorción de sustancias contaminantes durante los primeros días de operación, alcanzando una actividad estable, tal como se muestra en la figura 3.

FIGURA 3. Curva de desactivación típica de un catalizador de hidroprocesamiento.

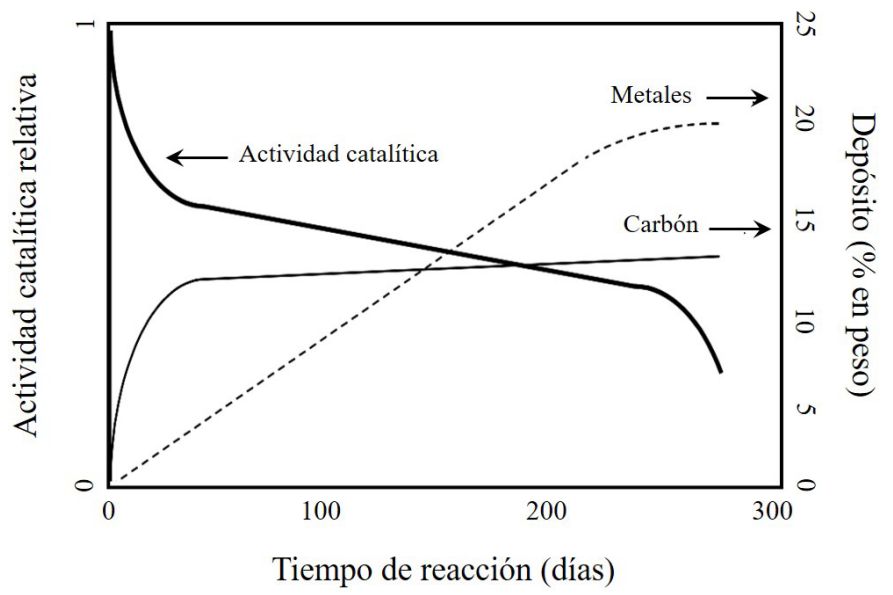

Fuente: Tomado de Vogelaar (2005). 
La actividad cae rápidamente debido al bloqueo de la estructura porosa causada por metales y coque; esto es lo que marca el tiempo de vida útil del catalizador que puede ser de hasta dos años de funcionamiento. Estos procesos de desactivación se aceleran durante el proceso de operación, pues en la práctica la temperatura de operación del reactor se incrementa gradualmente para compensar la pérdida de actividad catalítica. La desactivación inicial ofrece un reto para el mejoramiento del catalizador; hasta el 50\% de la actividad inicial se puede perder en los primeros días de operación del reactor. Posteriormente, cuando se alcanza la temperatura máxima de operación, se realizan procesos de regeneración, con la finalidad de reactivar la funcionalidad del catalizador.

\section{Desactivación por sinterización}

La sinterización consiste en el crecimiento del tamaño de las partículas metálicas, ocasionando disminución del área específica y, por consiguiente, disminución de la actividad catalítica. El proceso de sinterización es inducido por el incremento de la temperatura, iniciando a temperaturas superiores de los $500^{\circ} \mathrm{C}$. Además, este proceso puede ser acelerado por la presencia de vapor de agua (Fichtl et al., 2015).

Los mecanismos de sinterización son los siguientes:

a) Migración atómica.

b) Migración de cristales.

c) Transporte de vapor realizado a muy altas temperaturas.

Se considera también sinterización a la pérdida de área específica del soporte del catalizador, causado por el colapso del sistema poroso. Este proceso también se debe al incremento de la temperatura o a los procesos térmicos a los que se somete el catalizador durante su preparación.

Existen modelos conceptuales para predecir el crecimiento de las partículas metálicas, tal como se muestra en la figura 4.

La figura 4 muestra dos mecanismos de sinterización, migración atómica y migración de cristales. Ambos mecanismos generan tamaños de partículas en escala nanométrica que, dependiendo del tamaño de poro del soporte, los cristales pueden segregarse en la superficie externa del soporte o bloquear los poros, si la segregación se realiza mediante migración atómica en la superficie interna. En el caso de la segregación externa, existe la posibilidad de perder en su totalidad la actividad catalítica, si la corriente de alimentación presenta moléculas contaminantes como $\mathrm{H}_{2} \mathrm{~S}$, ya que los clusters metálicos estarían expuestos y no protegidos por la selectividad de forma del soporte.

Los factores que afectan el crecimiento del tamaño de las partículas metálicas son los siguientes (Argyle y Bartholomew, 2015): 
FIGURA 4. Modelo conceptual del proceso de sinterización.

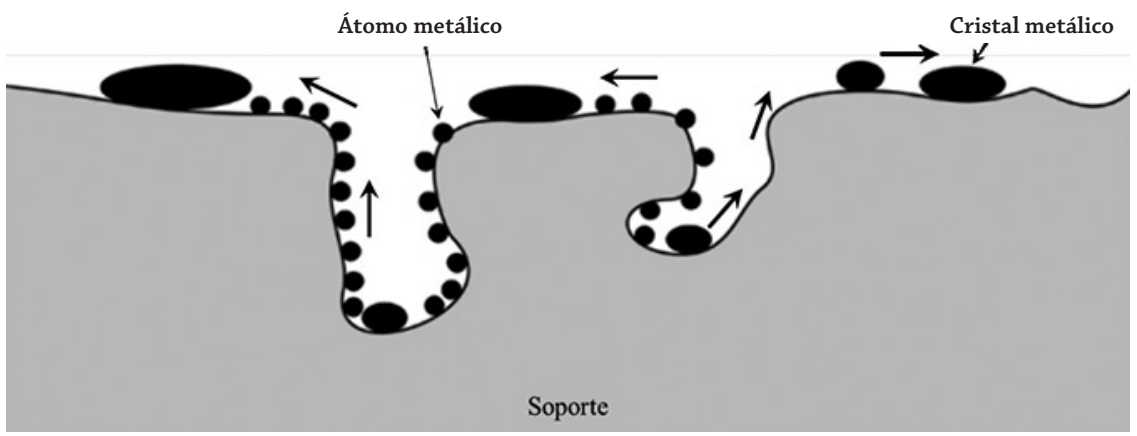

Fuente: Tomado de Bartholomew et al. (2006).
a) Temperatura.
b) Atmósfera.
c) Tipo de metal.
d) Tipo de soporte.
e) Presencia de promotores.
f) El tamaño de poro.

Por otro lado, el proceso opuesto a la sinterización es la redispersión, la cual consiste en formar óxidos metálicos volátiles o complejos cloro-metálicos anclados al soporte, siendo descompuestos mediante la reducción en pequeños cristales metálicos. Otra forma de redispersar consiste en formar películas delgadas que, posteriormente, serán fragmentadas en pequeños cristales durante el proceso de reducción (Argyle y Bartholomew, 2015).

\section{Desactivación por degradación química}

La desactivación por degradación química consiste en la desactivación del catalizador causada por las reacciones químicas siguientes:

a) Fase vapor y la superficie del catalizador. La pérdida de actividad se debe a la formación de una nueva fase. Este tipo de reacciones produce:

- $\quad$ Bulks inactivos. Por ejemplo, el $\mathrm{Rh}$ del catalizador Pt-Rh/ $\mathrm{Al}_{2} \mathrm{O}_{3}$ de los convertidores catalíticos de los autos reacciona con la $\gamma-\mathrm{Al}_{2} \mathrm{O}_{3}$ para formar $\mathrm{RhAl}_{2} \mathrm{O}_{4}$, que es inactivo. Otro ejemplo es la formación de $\mathrm{FeO}$ por la oxidación del catalizador $\mathrm{Fe} / \mathrm{K} /$ $\mathrm{Al}_{2} \mathrm{O}_{3}$ por la presencia de $\mathrm{O}_{2}$ y $\mathrm{H}_{2} \mathrm{O}$ en concentraciones de 50 ppm y 0.16 ppm, respectivamente, en la síntesis de amoniaco. Asimismo, la dealuminización de la zeolita Y durante el craqueo catalítico a alta temperatura, causa la destrucción de la zeolita (Argyle y Bartholomew, 2015). 
- Compuestos volátiles. Por ejemplo, la formación de $\mathrm{RuO}_{4}$ volátil del catalizador $\mathrm{Pd}-\mathrm{Ru} / \mathrm{Al}_{2} \mathrm{O}_{3}$ de los convertidores catalíticos de los autos, la formación de $\mathrm{Ni}(\mathrm{CO})_{4}$ del catalizador $\mathrm{Ni} / \mathrm{Al}_{2} \mathrm{O}_{3}$ en la metanación del $\mathrm{CO}$, la formación de $\mathrm{MoS}_{2}$, la formación de $\mathrm{CuCl} 2$ durante la síntesis del metanol, entre otros (Argyle y Bartholomew, 2015).

- Sinterización química a causa de especies adsorbidas. Este tipo de interacción guía la restructura de la superficie. Este modelo de sinterización se presenta aproximadamente a temperatura en el intervalo de $1 / 3$ a $\frac{1}{2}$ de la temperatura de fusión del metal o fase activa, es decir, cerca de la temperatura de Hütting (temperatura donde los átomos y defectos en la superficie del metal se vuelven móviles) o Tamman (temperatura donde los átomos en el seno del sólido presentan una cierta movilidad). Por caso, la formación de $\mathrm{Ni}(\mathrm{CO})_{4}$ volátil del catalizador $\mathrm{Ni} / \mathrm{Al}_{2} \mathrm{O}_{3}$ en la metanación del $\mathrm{CO}$ está seguida de su descomposición, formando cristales grandes de Ni. Otro ejemplo es la desactivación del catalizador $\mathrm{Ni} / \mathrm{Al}_{2} \mathrm{O}_{3}$ por la formación de $\mathrm{NiAl}_{2} \mathrm{O}_{4}$ a temperaturas cercanas a $450{ }^{\circ} \mathrm{C}$ (Argyle y Bartholomew, 2015).

b) Transformaciones en estado sólido. Digamos, la formación de $\mathrm{KAlO}_{2}$ durante la síntesis de amoniaco en la superficie catalítica de $\mathrm{Fe} / \mathrm{K} /$ $\mathrm{Al}_{2} \mathrm{O}_{3}$, la reducción de la fase activa $\mathrm{PdO}$ a Pd metálico del catalizador $\mathrm{PdO} / \mathrm{Al}_{2} \mathrm{O}_{3}$ a temperaturas superiores de $800{ }^{\circ} \mathrm{C}$, la formación de compuestos de $\mathrm{V}$ (IV) en la oxidación de $\mathrm{SO}_{2}$, entre otros (Argyle y Bartholomew, 2015).

\section{Desactivación por fallas mecánicas}

Los catalizadores comerciales son vulnerables a fallas mecánicas - esto debido a la forma que presentan -, tales como gránulos, esferas, extruidos y pellets. La desactivación del catalizador por fallas mecánicas se clasifica de las siguientes formas:

a) Crusing (aplastado). Este tipo de desactivación es causada por la cantidad de catalizador presente en algunos puntos del reactor, por lo general de lecho fijo, donde algunas partículas sólidas sostienen toneladas del catalizador.

b) Atrición o desgaste. Este tipo de desactivación se debe a la reducción de tamaño o rompimiento de las partículas del catalizador debido al choque entre ellas y con las paredes del reactor durante la operación de los reactores donde el catalizador presenta movimiento, tal es el caso de lecho móvil o fluidizado y el reactor de lodos, donde los polvos finos de catalizador son arrastrados por las corrientes del fluido. 
c) Erosión. Este tipo de desactivación se da en reactores tipo monolito donde se emplean altas velocidades del flujo de gas.

d) Fracturas. Este tipo de desactivación se debe al estrés térmico, mecánico o químico que presentan los catalizadores heterogéneos.

\section{Conclusiones}

Los catalizadores heterogéneos son ampliamente utilizados en diversos procesos de la industria de refinación del petróleo o en la producción de etileno mediante la deshidratación catalítica de etanol, utilizando como catalizadores las zeolitas HMOR y HZSM-5.

Dependiendo de la composición química que presenten las corrientes a la entrada del proceso, el catalizador sufrirá indudablemente disminución de la actividad catalítica, lo cual implica inversiones millonarias periódicas. Dicha disminución de actividad catalítica se debe a los diversos mecanismos de desactivación catalítica. Por lo cual, es necesario identificar el tipo de mecanismo que aplica a cada caso en particular. Los mecanismos de desactivación identificados son envenenamiento, ensuciamiento, sinterización, degradación térmica y fallas mecánicas, donde las causas son de naturaleza química, mecánica y térmica. Asimismo, se identificaron mecanismos lentos e irreversibles, como la quimisorción de azufre, y muy rápidos y reversibles, como el ensuciamiento por coque.

\section{Referencias}

Al-Khattaf, S. (2002). The influence of Y-zeolite unit cell size on the performance of FCC catalysts during gas oil catalytic cracking. Applied Catalysis A: General, 231(1-2): 293-306. https://doi.org/10.1016/S0926-860X(02)00071-6

Appleby, W. G., Gibson, J. W., Good, G. M. (1962). Coke formation in catalytic cracking. Industrial \& Engineering Chemistry Process Design and Development, 1(2): 102-110. https://doi.org/10.1021/i260002a006

Argyle, M. D., Bartholomew, C. H. (2015). Heterogeneous catalyst deactivation and regeneration: A review. Catalysts, 5(1): 145-269. https://doi.org/10.3390/ catal5010145

Augustine, S. M., Alameddin, G. N., Sachtler, W. M. H. (1989). The effect of Re, S, and $\mathrm{Cl}$ on the deactivation of $\mathrm{Pt} / \gamma-\mathrm{Al}_{2} \mathrm{O}_{3}$ reforming catalysts. Journal of Catalysis, 115(1): 217-232. https://doi.org/10.1016/0021-9517(89)90020-1

Bai, P., Etim, U. J., Yan, Z., Mintova, S., Zhang, Z., Zhong, Z., Gao, X. (2018). Fluid catalytic cracking technology: current status and recent discoveries on catalyst contamination. Catalysis Reviews, 61(3): 333-405. https://doi.org/10.1080/01 614940.2018.1549011

Bartholomew, C. H., Agrawal, P. K., Katzer, J. R. (1982). Sulfur poisoning of metals. Advances in Catalysis, 31: 135-242.

https://doi.org/10.1016/S0360-0564(08)60454-X 
Bartholomew, C. H., Farrauto, R. J. (2006). Catalyst deactivation: Causes, mechanisms, and treatment. En Fundamentals of industrial catalytic processes, 2a ed. EUA: John Wiley \& Sons, Inc. 260-336. https://doi.org/10.1002/9780471730071.ch5

Castañeda, R., Corma, A., Fornés, V., Martínez-Triguero, J., Valencia, S. (2006). Direct synthesis of a 9x10 member ring zeolite (Al-ITQ-13): A highly shape-selective catalyst for catalytic cracking. Journal of Catalysis, 238(1): 79-87.

Dufresne, P. (2007). Hydroprocessing catalysts regeneration and recycling. Applied Catalysis A: General, 322: 67-75. https://doi.org/10.1016/j.apcata.2007.01.013

Félix Flores, M. G. (2006). Simulación del proceso FCC: Caracterización de las corrientes de alimentación y productos del riser a través de la función de distribución gamma. Tesis de doctorado. Instituto Tecnológico de Celaya, México. http://www.iqcelaya.itc.mx/ richart/TesisDoctorado/2006\%20Felix\%20Flores.pdf

Fichtl, M. B., Schlereth, D., Jacobsen, N. Kasatkin, I., Schumann, J., Behrens, M., Schlögl, R., Hinrichsen, O. (2015). Kinetics of deactivation on $\mathrm{Cu} / \mathrm{ZnO} / \mathrm{Al}_{2} \mathrm{O}_{3}$ methanol synthesis catalysts. Applied Catalysis A: General, 502: 262-270. https://doi.org/10.1016/j.apcata.2015.06.014

Fuentes, S. (1996). Global overview of research in catalysis, Mexico. Applied Catalysis A: General, 142(1): 179-181. https://doi.org/10.1016/0926-860X(96)00011-7

Furimsky, E., Massoth, F. E. (1999). Deactivation of hydro processing catalysts. Catalysis Today, 52: 381-495. https://doi.org/10.1016/S0920-5861(99)00096-6

Hou, X., Qiu, Y., Yuan, E., Zhang, X., Liu, G. (2017). SO42-/TiO 2 promotion on HZSM-5 for catalytic cracking of paraffin. Applied Catalysis A: General, 537: 1223. https://doi.org/10.1016/j.apcata.2017.03.002

Ino, T., Al-Khattaf, S. (1996). Effect of unit cell size on the activity and coke selectivity of FCC catalysts. Applied Catalysis A: General, 142(1): 5-17. https://doi. org/10.1016/0926-860X(96)00018-X

Kallinikos, L. E., Bellos, G. D., Papayannakos, N. G. (2008). Study of the catalyst deactivation in an industrial gasoil HDS reactor using a mini-scale laboratory reactor. Fuel, 87(12): 2444-2449. https://doi.org/10.1016/j.fuel.2008.03.007

Larsson, A. C. (2007). Study of catalyst deactivation in three different industrial processes. Tesis de doctorado. Växjö University, Sweden. ISBN: 978-91-7636-533-5. https:// www.researchgate.net/publication/279641458_Study_of_Catalyst_Deactivation_in_ThreeDifferent_Industrial_Processes/related\#fullTextFileContent

Li, J. P., Yang, X. J., Ma, L., Yang, Q., Zhang, Y. H., Bai, Z. S., Fang, X. C., Li, L. Q., Gao, Y., Wang, H. L. (2016). The enhancement on the waste management of spent hydrotreating catalysts for residue oil by a hydrothermal-hydro cyclone process. Catalysis Today, 271: 163-171. https://doi.org/10.1016/j.cattod.2015.08.037

Liu, Z., Zhang, Z., Fan, H., Wang, Y., Liu, P., Yang, C. (2014). Research on the high activity of REY zeolite in fluid catalytic cracking reaction. Applied Petrochemical Research, 4: 385-388. https://doi.org/10.1007/s13203-014-0076-8

Mackor, E. L., Hofstra, A., Van der Waals, J. H. (1958). The basicity of aromatic hydrocarbons. Part 1. Unsubstituted polynuclear compounds. Transactions of the Faraday Society, 54: 66-83. https://doi.org/10.1039/TF9585400066

Martino, G., Boitiaux, J. P. (2002). Les catalyseurs: utilisations actuelles et évolu- 
tions prévisibles. L’Actualité Chimique, 257: 7-11. http://www.lactualitechimique.org/Quoi-de-neuf-en-catalyse

Menon, P. G. (1990). Coke on catalysts - harmful, harmless, invisible and beneficial types. Journal of Molecular Catalysis, 59(2): 207-220. https://doi.org/10.1016/03045102(90)85053-K

Naccache, C. (1985). Deactivation of acid catalysts. En Jacques Oudar y Henry Wise (cords.), Deactivation and poisoning of catalysts. Nueva York: Marcel Dekker, 185-203. ISBN-10: 0824773381

Qian, K., Tomczak, D. C., Rakiewicz, E. F., Harding, R. H., Yaluris, G., Cheng Cheng, W., Zhao, X., Peters, A.W. (1997). Coke formation in the fluid catalytic cracking process by combined analytical techniques. Energy Fuels, 11(3): 596-601. https://doi.org/10.1021/ef960204u

Seki, H., Yoshimoto, M. (2001). Deactivation of HDS catalyst in two-stage RDS process II. Effect of crude oil and deactivation mechanism. Fuel Processing Technology, 69(3): 229-238. https://doi.org/10.1016/S0378-3820(00)00143-0

Song, C. (1999). A proposed new concept for design of sulfur-resistant noble metal catalysts based on shape-selective exclusion and hydrogen spillover. En Chunshan Song, Juan M. Garcés y Yoshihiro Sugi (cords.), Shape-selective catalysis. ACS Symposium Series, 381-389. https://doi.org/10.1021/bk-2000-0738.ch027

Song, C., Schmitz, A. D. (1997). Zeolite-supported Pd and Pt catalysts for low-temperature hydrogenation of naphthalene in the absence and presence of benzothiophene. Energy Fuels, 11(3): 656-661. https://doi.org/10.1021/ef960179s

Téllez-Romero, J. G., Cuevas-García, R., Ramírez, J., Castillo-Villalón, P., ContrerasBárbara, R., Salcedo-Luna, M. C., Puente-Lee, R. I. (2015). Simultaneous naphthalene and thiophene hydrogenation over $\mathrm{Ni}(\mathrm{X})-\mathrm{Pt} / \mathrm{HMOR}$ catalysts. Catalysis Today, 250: 12-20. https://doi.org/10.1016/j.cattod.2014.08.032

Vogelaar, B. M. (2005). Deactivation of hydroprocessing catalysts: New insights in catalyst structure, activity and stability. Tesis de doctorado. TU Delft, Delft University of Technology.

http://resolver.tudelft.nl/uuid:1f5f31e7-b83a-4b49-b622-bb461ae19d30

Vogt, E. T., Weckhuysen, B. M. (2015). Fluid catalytic cracking: recent developments on the grand old lady of zeolite catalysis. Chemical Society Reviews, 44: 73427370. https://doi.org/10.1039/C5CS00376H

Yasuda, H., Yoshimura, Y. (1997). Hydrogenation of tetralin over zeolite-supported Pd-Pt catalysts in the presence of dibenzothiophene. Catalysis Letters, 46(1-2): 43-48. https://doi.org/10.1023/A:1019021224505

Zakari Yusuf, A., Mandafiya John, Y., Olufemi Aderemi, B., Patel, R., Mujtaba, I. M. (2019). Modelling, simulation and sensitivity analysis of naphtha catalytic reforming reactions. Computers \& Chemical Engineering, 130: 1-16. https://doi. org/10.1016/j.compchemeng.2019.106531

Zhou, J., Zhao, J., Zhang, J., Zhang, T., Ye, M., Liu, Z. (2020). Regeneration of catalysts deactivated by coke deposition: A review. Chinese Journal of Catalysis, 41(7): 1048-1061. https://doi.org/10.1016/S1872-2067(20)63552-5 